\title{
NUMERICAL ESTIMATION OF EFFECTIVE ELASTIC MODULI OF SYNTACTIC FOAMS REINFORCED BY SHORT GLASS FIBERS
}

\author{
${ }^{\#}$ WEI YU, MENG QIAN, XI LIANG, HUIJIAN LI \\ Key Laboratory of Mechanical Reliability for Heavy Equipments and Large Structures of Hebei Province, \\ Yanshan University, Qinhuangdao 066004, China \\ "E-mail: yuweichn@163.com
}

Submitted September 17, 2015; accepted January 27, 2016

\begin{abstract}
Keywords: Syntactic foams, Glass fiber, Hollow glass microsphere, Representative volume elements
The mechanical properties of hollow glass microspherelepoxy resin syntactic foams reinforced by short glass fibers are studied using representative volume elements. Both the glass fibers and the hollow glass microspheres exhibit random arrangement in the epoxy resin. The volume fraction and wall thickness of hollow glass microspheres and the volume fraction of glass fibers are considered as parameters. It is observed that the elastic modulus values of syntactic foams decrease with the increase of microsphere volume fraction when the microsphere relative wall thickness is lower. However, it increases with the increase of microsphere volume fraction when the relative wall thickness exceeds a critical value. The elastic modulus value goes through a maximum when the relative wall thickness is around 0.06 at $25 \%$ volume fraction of microspheres. The addition of glass fibers reduces the critical wall thickness values of the microspheres and increases the mechanical properties of the composites. The highest stress lies on the equatorial plane perpendicular to the loading direction. Adding fibers reduces the large stress distribution areas on the microspheres, and the fibers aligned with the loading direction play an important load-bearing role.
\end{abstract}

\section{INTRODUCTION}

Hollow particles filling a polymeric matrix form composites called syntactic foams. In recent years, syntactic foams have been widely studied due to their low weight, low moisture absorption, high specific mechanical properties, and good impact performance [13]. Epoxy resins, polyurethane, polyethylene, and several other polymers are commonly used as binders. The hollow particles called microspheres or microballoons are made of polymers, glass, ceramic, carbon, and even metals. Syntactic foams have been widely used in engineering, e.g. automotive industry, sports equipment, marine equipment and space structures [4-6].

Experimental studies have shown that the properties of syntactic foams change with the volume fraction, wall thickness and size of hollow particles. Adding a higher volume fraction of hollow particles can greatly reduce the density of syntactic foams, which can make them appropriate for use in automotive, aerospace and marine structures. However, the strength and stiffness of composites may greatly decrease with higher volume fractions of hollow particles [7-9]. Many studies reported in the literature show that adding fibers in syntactic foams is one of the methods to enhance the mechanical properties of composites [10-18]. Several studies on glass fiber reinforced polymer composites have been published earlier [19-24]. Carbon nanofibers and nanoclay have also been used as reinforcements in some studies $[6,25$, $26]$. The effect of fiber volume fraction and length on mechanical and thermal properties has been studied by Wouterson et al. [27]. Their experimental studies show that the tensile strength, flexural strength and elastic modulus increase with the increase of fiber volume fraction.

However, the effect of hollow sphere wall thickness and diameter on the mechanical properties of syntactic foams is difficult to consider in experimental studies. A few theoretical methods have shown the effect of hollow sphere volume fraction and wall thickness on the elastic modulus of syntactic foams [28, 29]. However, theoretical researches of syntactic foams with fiber reinforcement are rare. Numerical methods are effective tools to investigate the effect of particle volume fraction and wall thickness on the mechanical behavior of syntactic foams [30-32]. Nguyen and Gupta [33] have analyzed the effect of fiber reinforcement on the properties of syntactic foams. In all these works the distribution of particles and fibers is assumed to be uniform. In the present study, representative volume elements are used to investigate the elastic modulus and stress distribution in the matrix of glass fibers and hollow glass microspheres randomly filling epoxy resin. The volume fraction and wall thickness of the hollow glass microspheres and three volume fractions of glass fibers are considered in these numerical models. 


\section{EXPERIMENTAL}

\section{Analysis parameters}

In composites reinforced with discontinuous short fibers or particles, the reinforcements are usually not lined up, but their position and orientation are random and uniform. The model considered in this paper is based on these facts. In this study, six different volume fractions and six different wall thicknesses of hollow glass microspheres, as well as three different volume fractions of glass fibers are used. It has been shown in the literature [34] that the error is less than $5 \%$ when the model length is more than twice larger than the particle diameter. In the representative volume element (REV) used here, the dimensions are $180 \times 180 \times 180 \mu \mathrm{m}$, and particles with diameter $50 \mu \mathrm{m}$ and fibers with diameter $16 \mu \mathrm{m}$ and length $80 \mu \mathrm{m}$, are randomly embedded in it, shown in Figure 1a. Random sequential adsorption is used to generate the models [35-37]. Figure $1 \mathrm{~b}$ and Figure 1c show the representative volume element and its meshed model, respectively. The meshing of the representative volume element is denser close to the filler and less dense in the matrix far away from the filler. And the 10 nodes tetrahedral isoparameter element is adopted in this paper. Here, $V_{\mathrm{s}}$ is the volume fraction of hollow glass microspheres, and $V_{\mathrm{f}}$ is the volume fraction of glass fibers.

In total, 185 three-dimensional solid elements are used to mesh the matrix and glass fibers, and 181 shell elements are used to mesh the hollow glass microspheres. The material properties used for the calculations are shown in Table 1. This set of parameters is selected for epoxy resin syntactic foams studied in many works. There are only small differences of elastic modulus between glass microspheres and glass fibers. Of course, both the hollow glass microspheres and the glass fibers are assumed to be linearly elastic and isotropic. The interfaces of microsphere-epoxy and fiber-epoxy are assumed to be perfect. The elastic modulus is calculated by applying $1 \%$ compressive strain along the y direction on the model. All the simulations are performed with ANSYS 14.0.
Table 1. Material properties.

\begin{tabular}{lccc}
\hline & $\begin{array}{c}\text { Elastic modulus } \\
E(\mathrm{GPa})\end{array}$ & $\begin{array}{c}\text { Poisson's ratio } \\
\mu\end{array}$ & $\begin{array}{c}\text { Density } \\
\rho\left(\mathrm{g} \cdot \mathrm{cm}^{-3}\right)\end{array}$ \\
\hline Epoxy resin & 3 & 0.35 & 1.16 \\
\hline $\begin{array}{l}\text { Hollow glass } \\
\text { microsphere }\end{array}$ & 70 & 0.20 & 2.18 \\
\hline Glass fiber & 73 & 0.20 & 2.18 \\
\hline
\end{tabular}

In these models, the RVE contains numerous glass microspheres and glass fibers. The RVE corresponds to a cube cut from the material. Free boundary conditions are selected for saving calculation time. The surfaces through the origin point are set as simply supported, the other three surfaces set as free. In the process of loading, the surface needs to remain parallel to the uniform deformation, so that the three free surfaces are coupling correspondingly. Figure 2 compares the relative modulus $\left(E / E_{\mathrm{m}}\right)$ of syntactic foams containing hollow glass microspheres obtained from the free boundary assumed in this study, the periodic boundary assumed in the literature [35], and theoretical results calculated according to the mixture law

$$
E=E_{\mathrm{s}} \eta V_{\mathrm{s}}+E_{\mathrm{m}}\left(1-V_{s}\right),
$$

where, $E$ is the modulus of composite, $E_{\mathrm{m}}$ the modulus of the epoxy matrix, $E_{\mathrm{s}}$ the modulus of the hollow glass microsphere, and $\eta$ the relative wall thickness which is defined as the ratio of wall thickness to the radius of the hollow glass microsphere. The relative wall thickness $\eta$ is added to the formula in order to represent the hollowness of the glass microsphere. From Figure 2, it is evident that the results based on the two kinds of boundary conditions are very close. Actually, there is a maximum deviation of only $4.8 \%$ in the relative modulus values at $30 \%$ volume fraction of microspheres. The theoretical values are intermediate when the relative wall thickness is 0.02 , and slightly higher than both other values when the relative wall thickness is 0.08 . Therefore, the boundary condition used in this paper is likely to be more realistic when the relative wall thickness value is lower.

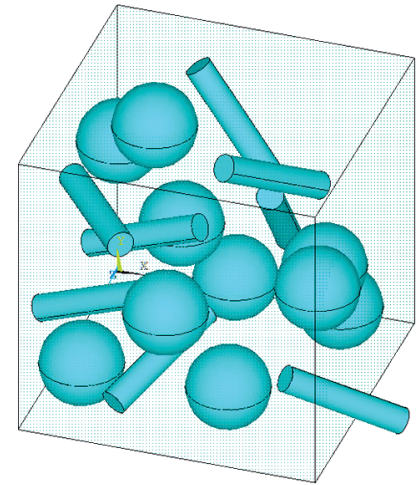

a)

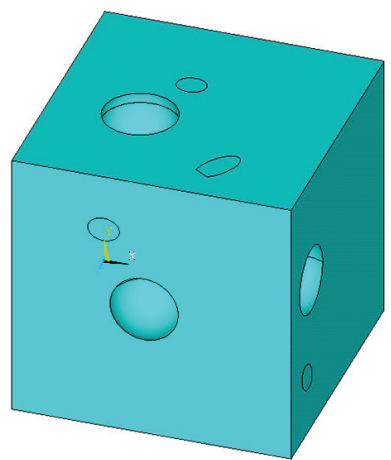

b)

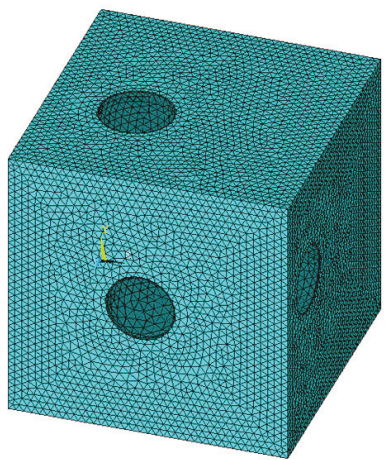

c)

Figure 1. Example of a RVE and its meshed model $\left(V_{\mathrm{s}}=10 \%, V_{f}=2 \%\right)$. 


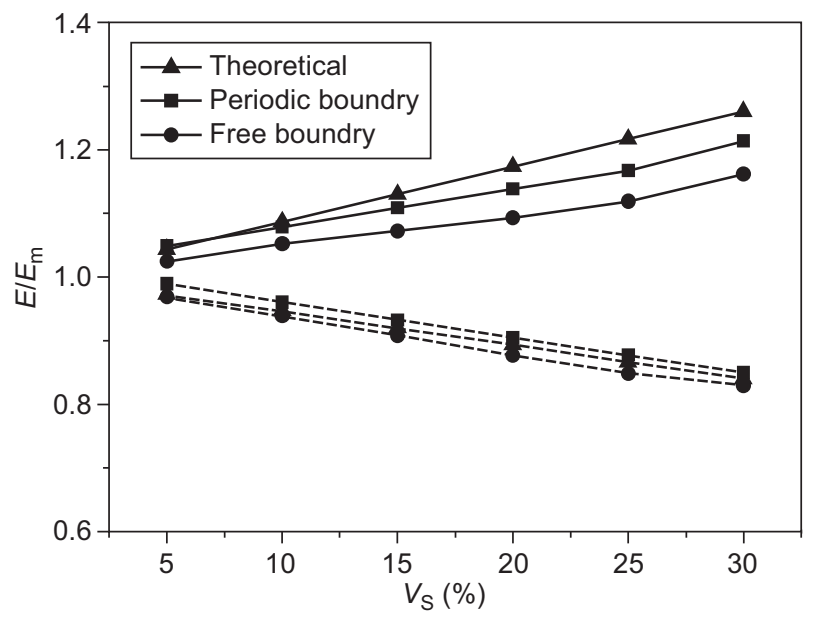

Figure 2. Relative modulus of syntactic foams, $\eta=0.02$ (dashed lines), and $\eta=0.08$ (solid lines).

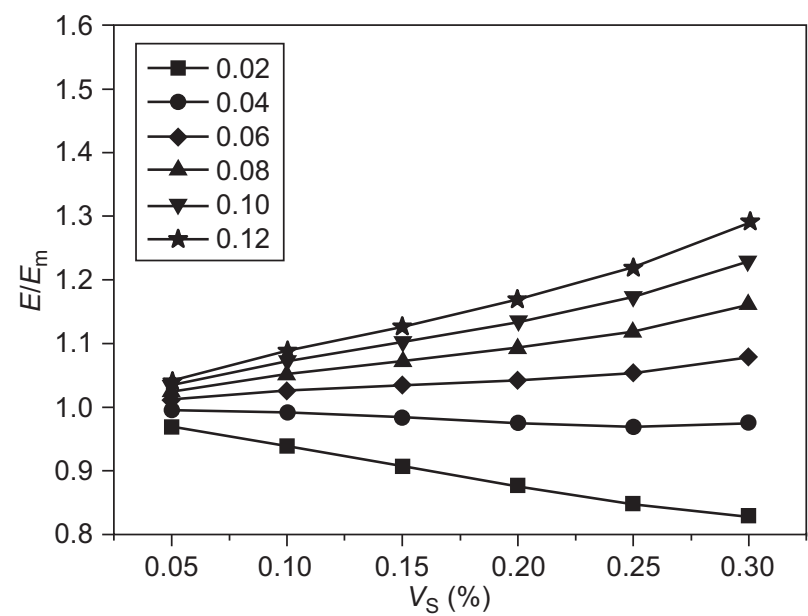

a) $V_{f}=0 \%$

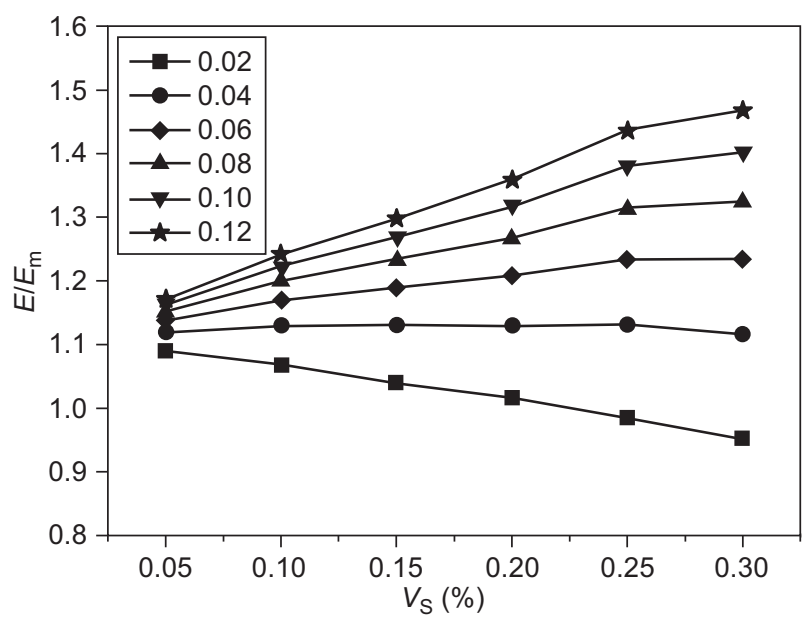

c) $V_{f}=4 \%$

\section{RESULTS AND DISCUSSION}

The volume fraction, relative wall thickness of hollow glass microspheres and glass fiber volume fraction are considered as influencing factors in this paper. Six relative wall thicknesses $(0.02-0.12)$, six microsphere volume fractions ( $5-30 \%$ ), and three glass fiber volume fractions have been selected. Figure 3 shows the influence of microsphere volume fractions on the relative modulus. The figure shows that the modulus decreases with the increase of microsphere volume fraction when the relative wall thickness values is 0.02 , but the modulus increases with the increase of microsphere volume fraction when the relative wall thickness values more than 0.06. It indicates that the microsphere wall thickness is a significant factor affecting the composite's modulus. In addition, Figure $3 b-d$, containing glass fibers, shows that the composite's modulus exhibits a significant change when the microsphere volume fraction is $25 \%$, at which an

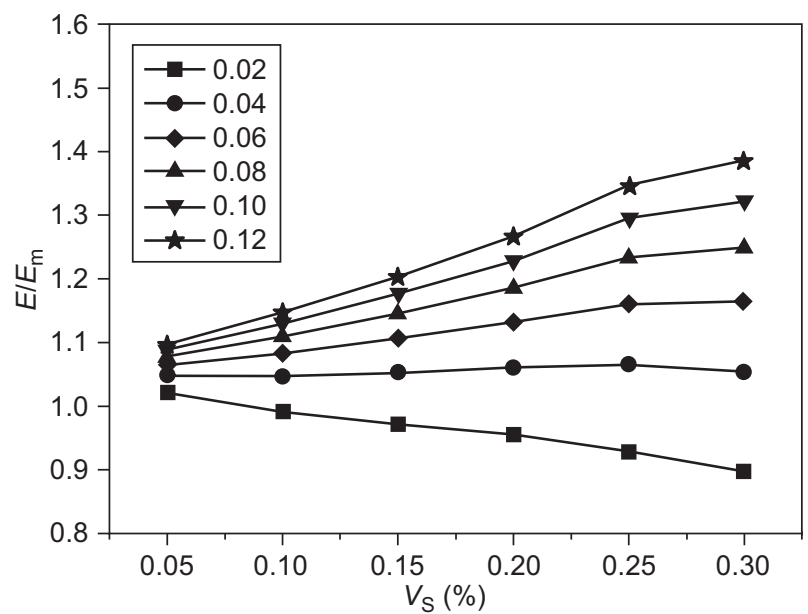

b) $V_{f}=2 \%$

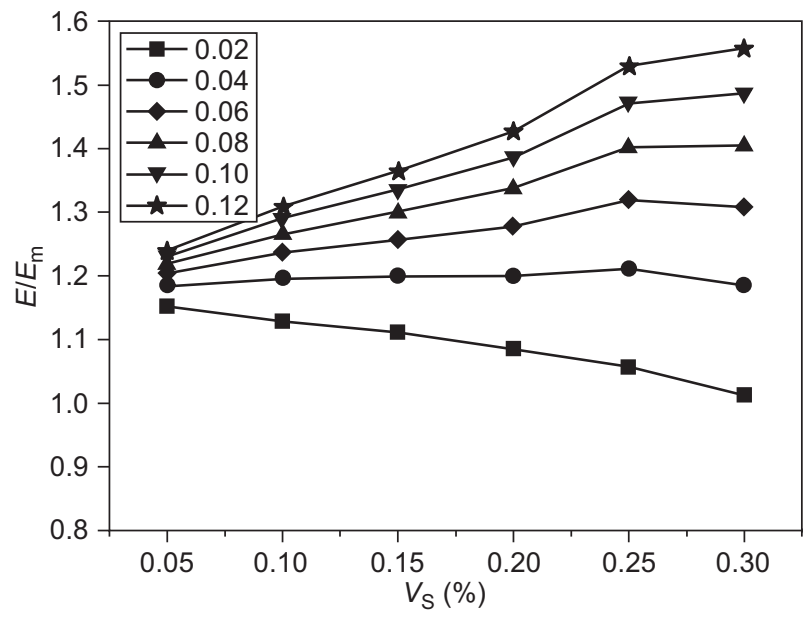

d) $V_{f}=6 \%$

Figure 3. Dependence of the relative elastic modulus on the microsphere volume fraction. 
obvious kink appears which becomes a maximum when the relative wall thickness is around 0.06 . The higher the glass fiber volume fraction, the more obvious is the turning point. The reason for this phenomenon is supposed to be the particle and fiber clustering when the microsphere volume fraction more than $25 \%$. At this point, the fibers affect the stress distribution of the microsphere. However, the effect reduces when the microsphere wall thickness is much smaller or much larger than 0.06 .

Figure 4 shows the effect of the relative wall thickness of microspheres on the composite's modulus. The figure shows that the relative modulus values increase with the relative wall thickness of microspheres for microsphere volume fractions from 5 to $30 \%$. It is obvious that the higher the microsphere volume fraction, the steeper is the dependence. It also can be seen that the curves exhibit a critical relative wall thickness at 0.04 for composite with fibers and slightly higher (0.05) for composites without fibers. Above this critical relative wall thickness value the elastic modulus increases with increasing microsphere volume fraction, but below this critical value the elastic modulus increases with decreasing microsphere volume fraction. This fact should be considered in material design. Figure 5 shows the change of the relative modulus with the fiber volume fraction. Relative wall thicknesses of $0.02,0.06$ and 0.1 have been selected in the figures. It is observed that the elastic modulus of the composites obviously increases by adding glass fibers.

Figure 6 compares the Von-Mises stress distribution with $V_{\mathrm{s}}=10 \%$ and $V_{\mathrm{f}}=2 \%$. The figures show that the microspheres bear larger stress state when the relative wall thickness is lower, and the highest stress lies on the equatorial plane perpendicular to the loading direction (except for the incomplete microspheres). The reason is that the thin wall thickness of the microsphere weakens the mechanical properties. However, this phenomenon is relieved with the increase of wall thickness, as shown in Figure $6 \mathrm{c}-\mathrm{d}$. When the relative wall thickness is increased, the location of maximum von-Mises stress gradually moves to the fiber, indicating that the microsphere relative wall thickness is an important
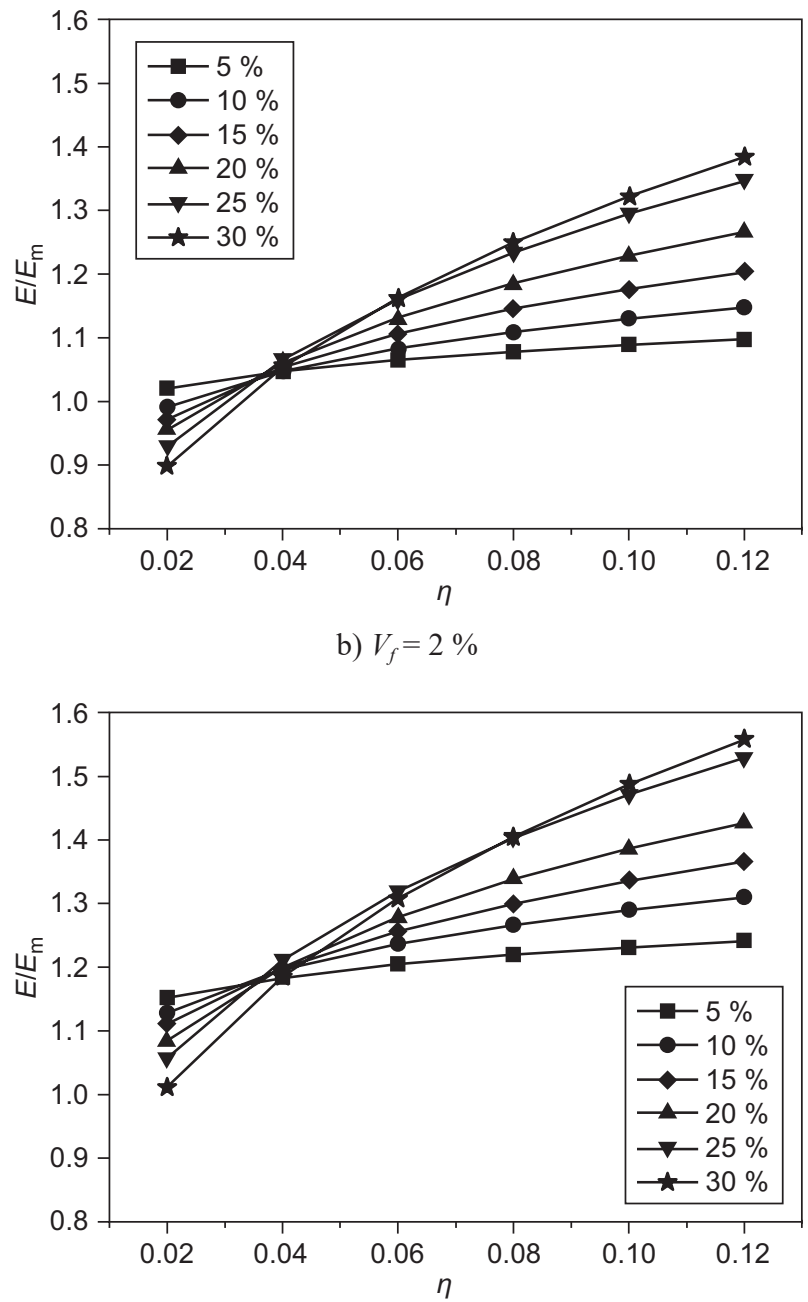

b) $V_{f}=2 \%$

d) $V_{f}=6 \%$

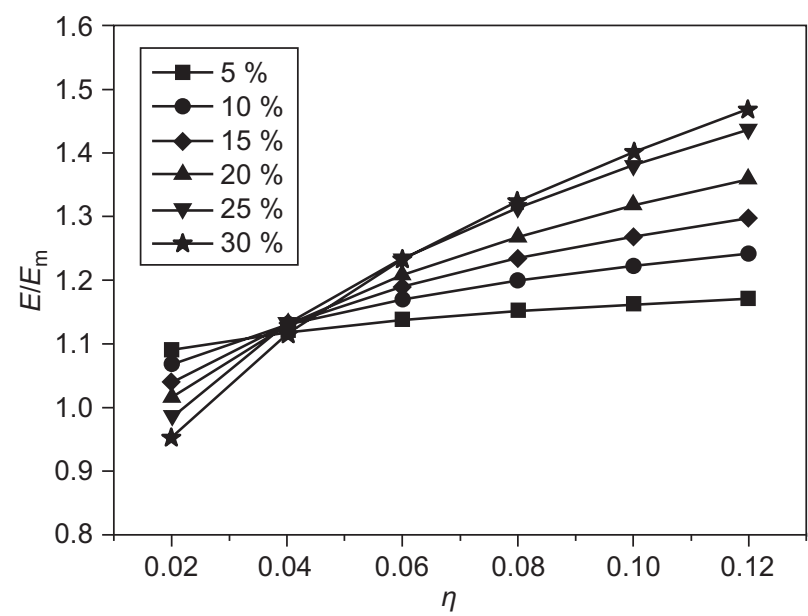

c) $V_{f}=4 \%$

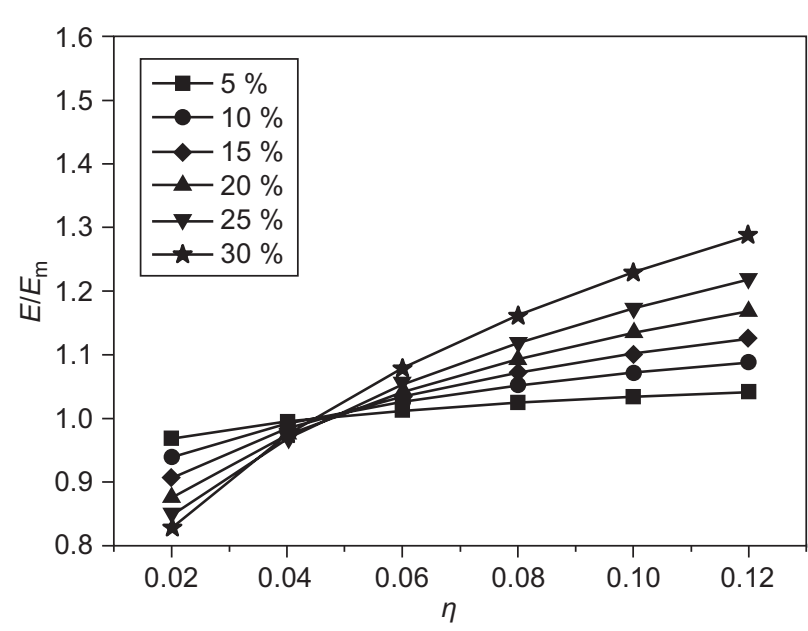

a) $V_{f}=0 \%$

Figure 4. Dependence of the relative elastic modulus on the relative wall thickness of the microspheres. 


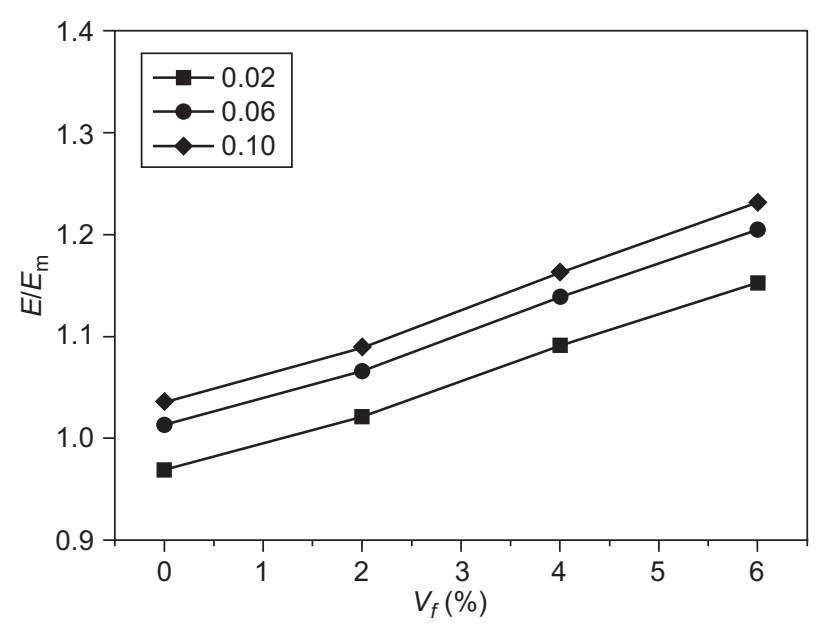

a) $V_{s}=5 \%$

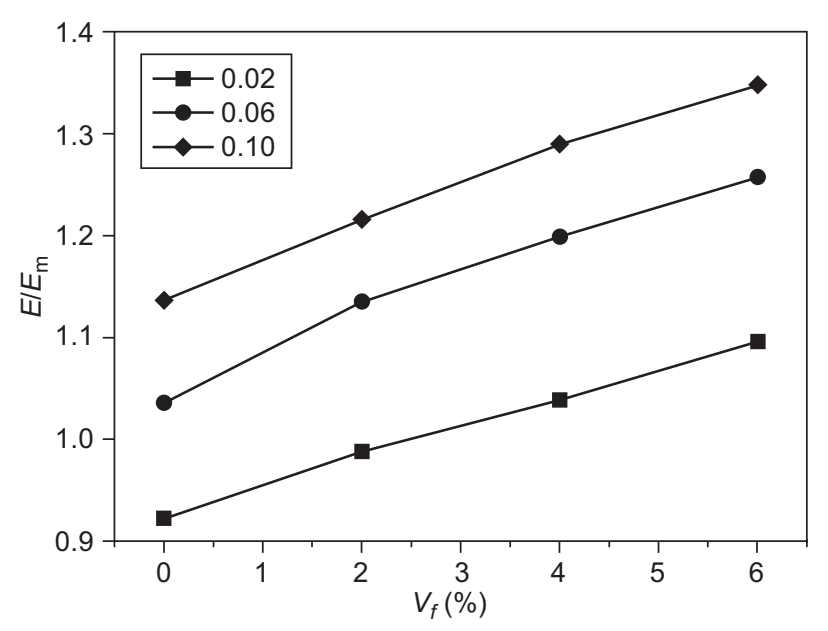

b) $V_{s}=20 \%$

Figure 5. Dependence of the relative elastic modulus on the fiber volume fraction.

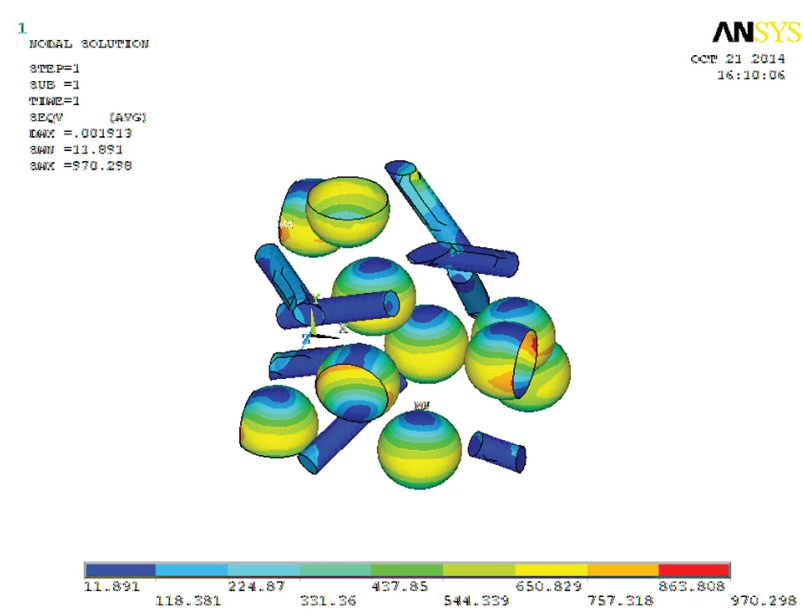

a) $\eta=0.02$
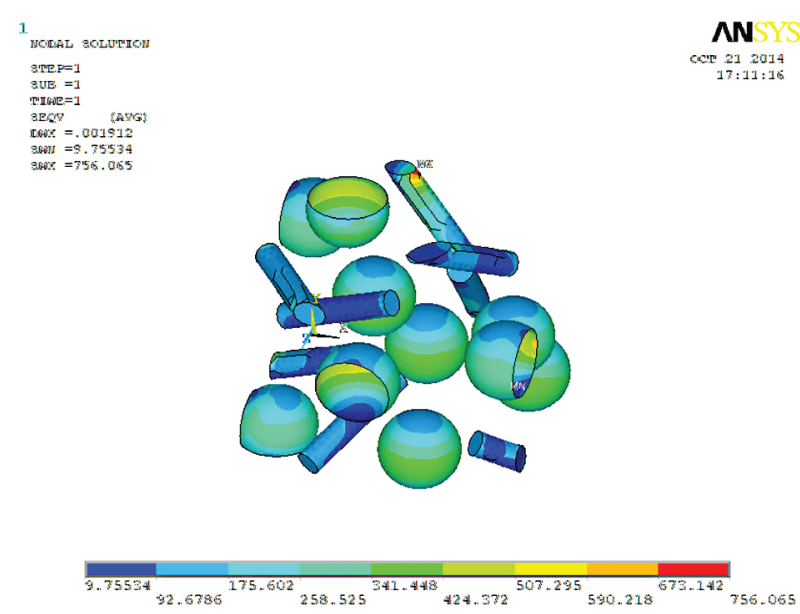

c) $\eta=0.08$

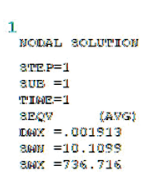

NN

$\underset{\cos 2: 21: 2014}{17: 03: 28}$
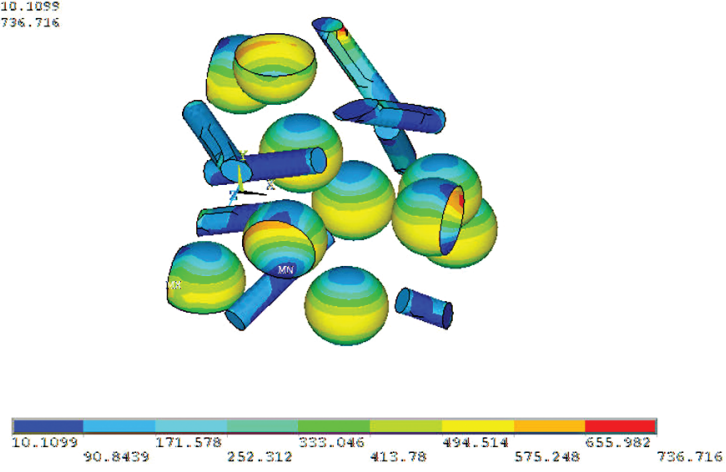

b) $\eta=0.04$
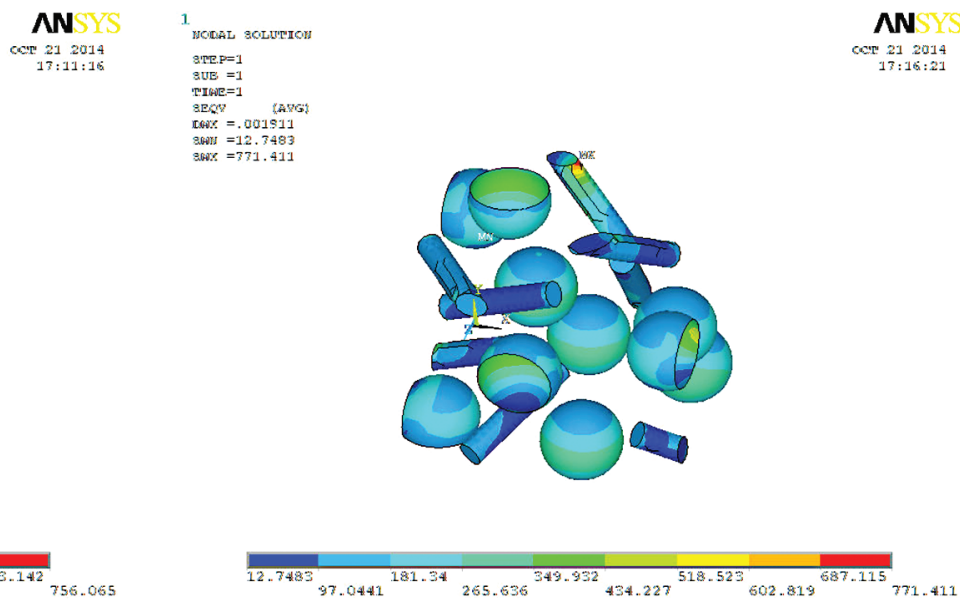

d) $\eta=0.12$

Figure 6. Von-Mises stress distribution with $V_{\mathrm{s}}=10 \%$ and $V_{\mathrm{f}}=2 \%$. 
parameter of the composite's mechanical properties.

Figure 7 shows the Von-Mises stress distribution of microspheres and fibers with microsphere relative wall thickness $\eta=0.02$. It is observed in Figure 7 that the maximum von-Mises stress also lies on the equatorial plane perpendicular to the loading direction (except for the incomplete microspheres), and the value changes only a little when the microsphere volume faction increase. It can be seen from Figure $7 b$ that the maximum von-Mises stress lies on the equatorial plane perpendicular to the loading direction of the complete microspheres, but not for the incomplete microspheres. The reason is that there are the fibers along the direction near that microsphere leading to microsphere's stress distribution changes. The figures also show that the stress on the glass fiber along the direction of loading is greater than that perpendicular to the loading direction.

Figure 8 shows the Von-Mises stress distribution comparison of syntactic foams with fiber and without fiber at microsphere volume fraction $V_{\mathrm{s}}=10 \%$. It is obvious that the fiber plays an important load-bearing role. The larger stress distribution areas on microspheres are reduced by adding fibers. The location of maximum von-Mises stress lies on the fiber edge aligned with the loading direction. This confirms that adding fibers is beneficial to the improvement of the mechanical performance of the syntactic foams.

\section{CONCLUSIONS}

The effective elastic modulus of syntactic foams reinforced by short glass fibers is calculated in the present study using representative volume elements, with short glass fibers and hollow glass microspheres randomly filling an epoxy resin matrix. A simple mixture law to estimate the modulus of syntactic foams is used with a relative wall thickness parameter. The results are

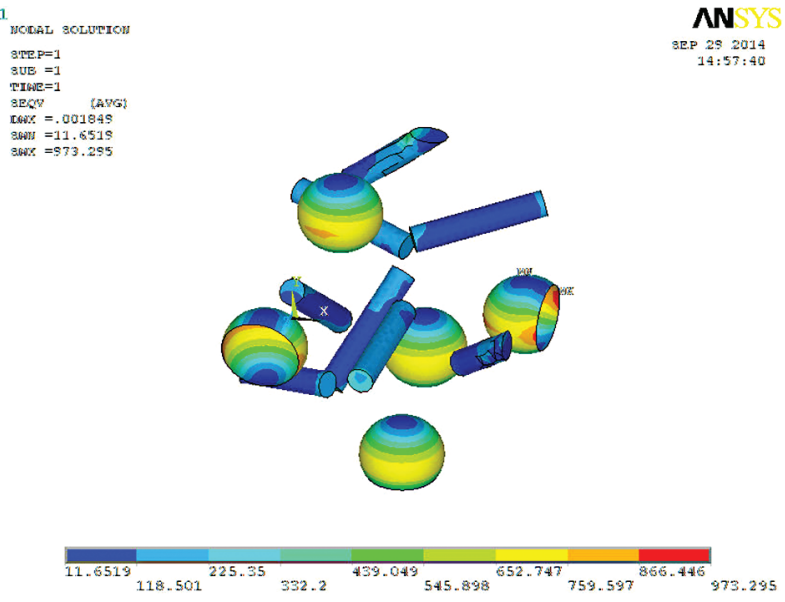

a) $V_{s}=5 \%$
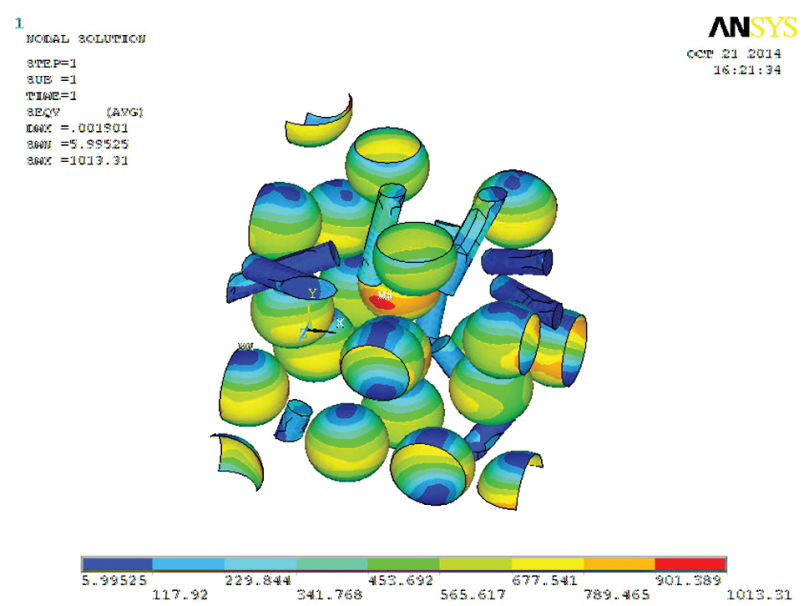

b) $V_{s}=20 \%$

Figure 7. Von-Mises stress distribution with fiber $V_{f}=2 \%$ and microsphere $\eta=0.02$.

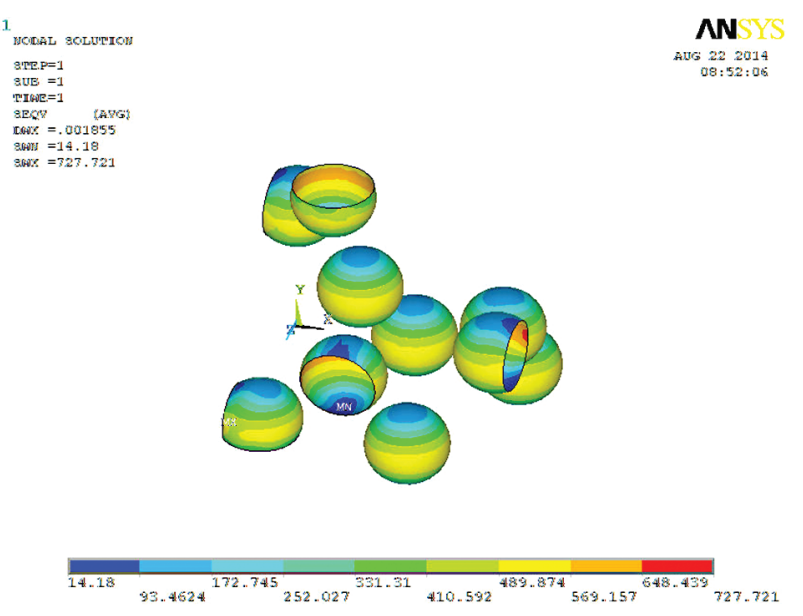

a) $V_{f}=0 \%$
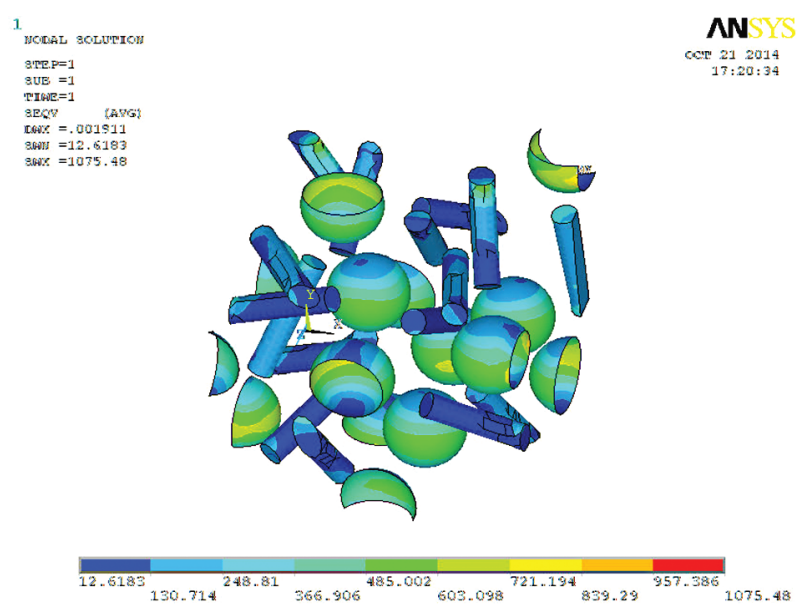

b) $V_{f}=4 \%$

Figure 8. Von-Mises stress distribution with $V_{s}=10 \%$ and $\eta=0.04$. 
close to the simulated results with periodic boundary. The volume fraction and wall thickness of hollow glass microspheres and the volume fraction of glass fibers are considered as the parameters in this study. It is found that the elastic modulus values of syntactic foams decreases with the increase of microsphere volume fraction when the relative wall thickness is less than a critical value $(0.04-0.05)$. However, it increases with the increase of microsphere volume fraction when the relative wall thickness is more than a critical value. The addition of glass fibers decreases the microsphere critical wall thickness values slightly. The composite's elastic modulus exhibits a significant change when the microsphere volume fraction is $25 \%$, at which an obvious kink appears which becomes a maximum when the relative wall thickness is around 0.06 . The reason for this phenomenon is supposed to be the particle and fiber clustering when the microsphere volume fraction more than $25 \%$.

The microspheres bear larger stresses when the relative wall thickness is lower, and the highest stress lies on the equatorial plane perpendicular to the loading direction (except for the incomplete microspheres). However, the location of maximum von-Mises stress gradually moves to the fiber by increasing the relative wall thickness. It is observed that adding glass fibers increases the elastic modulus values of composites in all the models, and the larger stress distribution areas on microspheres are reduced by adding fibers. The fibers aligned with the loading direction play an important loadbearing role, indicating that adding fibers is beneficial to the improvement of the mechanical performance of syntactic foams.

\section{Acknowledgments}

The research work is supported by the Natural Science Foundation of Hebei Province (No. A2014203051), and the Key Project of Science and Technology of Hebei Higher School (No. ZH2012011), and the Science and Technology Research and Development Plan of Qinhuangdao (No. 201401A054).

\section{REFERENCES}

1. Gupta N., Woldesenbet E. (2003): Hygrothermal studies on syntactic foams and compressive strength determination. Composite Structures, 61(4), 311-320. doi:10.1016/S02638223(03)00060-6

2. Bardella L., Genna F. (2001): On the elastic behavior of syntactic foams. International Journal of Solids and Structures, 38(40), 7235-7260. doi:10.1016/S0020-7683(00) 00228-6

3. Kim H.S., Khamis M.A. (2001): Fracture and impact behaviours of hollow micro-sphere/epoxy resin composites. Composites Part A: Applied Science and Manufacturing,
32(9), 1311-1317. doi:10.1016/S1359-835X(01)00098-7

4. Lu Z.X. (2004): Adv. Mech. 34, 341

5. Gupta N., Woldesenbet E., Mensah P. (2004): Compression properties of syntactic foams: effect of cenosphere radius ratio and specimen aspect ratio. Composites Part A: Applied Science and Manufacturing, 35(1), 103-111. doi:10.1016/j. compositesa.2003.08.001

6. Dimchev M., Caeti R., Gupta N. (2010): Effect of carbon nanofibers on tensile and compressive characteristics of hollow particle filled composites. Materials \& Design, 31(3), 1332-1337. doi:10.1016/j.matdes.2009.09.007

7. Swetha C., Kumar R. (2011): Quasi-static uni-axial compression behaviour of hollow glass microspheres/epoxy based syntactic foams. Materials \& Design, 32(8), 41524163. doi:10.1016/j.matdes.2011.04.058

8. Tagliavia G., Porfiri M., Gupta N. (2010): Analysis of flexural properties of hollow-particle filled composites. Composites Part B: Engineering, 41(1), 86-93. doi:10.1016/j.compositesb.2009.03.004

9. Wouterson E.M., Boey F.Y.C., Hu X., Wong S.C. (2005): Specific properties and fracture toughness of syntactic foam: Effect of foam microstructures. Composites Science and Technology, 65(11), 1840-1850. doi:10.1016/j.compscitech.2005.03.012

10. Karthikeyan C.S., Sankaran S., Jagdish K.M.N., Kishore (2001): Processing and compressive strengths of syntactic foams with and without fibrous reinforcements. Journal of applied polymer science, 81(2), 405-411. doi:10.1002/ app. 1452

11. Karthikeyan C.S., Sankaran S., Kishore (2004): Mater. Lett. 58, 995 Elastic behaviour of plain and fibre-reinforced syntactic foams under compression. Materials Letters, 58(6), 995-999. doi:10.1016/j.matlet.2003.08.012

12. Karthikeyan C.S., Sankaran S., Kishore (2005): Flexural Behaviour of Fibre-Reinforced Syntactic Foams. Macromolecular Materials and Engineering, 290(1), 60-65. doi:10.1002/mame.200400177

13. Alonso M.V., Auad M.L., Nutt S. (2006): Short-fiberreinforced epoxy foams. Composites part A: Applied Science and manufacturing, 37(11), 1952-1960. doi:10.1016/j. compositesa.2005.12.011

14. Huang Y.J., Wang C.H., Huang Y.L., Guo G.J., Nutt S.R. (2010): Enhancing specific strength and stiffness of phenolic microsphere syntactic foams through carbon fiber reinforcement. Polymer Composites, 31(2), 256-262. doi:10.1002/pc.20795

15. Ferreira J.A.M., Capela C., Costa J.D. (2010): A study of the mechanical behaviour on fibre reinforced hollow microspheres hybrid composites. Composites Part A: Applied Science and Manufacturing, 41(3), 345-352. doi:10.1016/j.compositesa.2009.10.018

16. Ferreira J.A.M., Salviano K., Costa J.D., Capela C. (2010): Fatigue behaviour in hybrid hollow microspheres/fibre reinforced composites. Journal of Materials science, 45(13), 3547-3553. doi:10.1007/s10853-010-4397-4

17. Zegeye E., Ghamsari A.K., Woldesenbet E. (2014): Mechanical properties of graphene platelets reinforced syntactic foams. Composites Part B: Engineering, 60, 268-273. doi:10.1016/j.compositesb.2013.12.040

18. Wang L.J., Zhang J., Yang X., Zhang C., Gong W., Yu J. (2014): Flexural properties of epoxy syntactic foams reinforced by fiberglass mesh and/or short glass fiber. Materials \& Design, 55, 929-936. doi:10.1016/j. 
matdes.2013.10.065

19. Cotgreave T.C., Shortall J.B. (1977): The mechanism of reinforcement of polyurethane foam by high-modulus chopped fibres. Journal of Materials Science, 12(4), 708717. doi:10.1007/BF00548161

20. Morimoto K., Suzuki T. , Yosomiya R. (1984): Adhesion between glass-fiber and matrix of glass-fiber reinforced rigid polyurethane foam under tension. Polymerplastics technology and engineering, 22(1), 55-76. doi: 10.1080/03602558408070031

21. Yosomiya R., Morimoto K. (1985): Effect of interaction between fiber and matrix on impact properties of glass fiber reinforced rigid polyurethane foam. PolymerPlastics Technology and Engineering, 24(1), 11-26. doi:10.1080/03602558508070055

22. Li G.Z., Yu Y.Z. (1997): Approach of reinforced mechanism of the glass fibers on the PUR foam plastics. Polym. Mater. Sci. Eng., 13, 130-133.

23. Lu Z.X., Wang J.H., Xie R.Z., Tian C.J., Li H.X. (1999): Acta. Mater. Compos. Sinica 16, 39.

24. Shen H.B., Nutt S. (2003): Mechanical characterization of short fiber reinforced phenolic foam. Composites Part A: applied science and manufacturing, 34(9), 899-906. doi:10.1016/S1359-835X(03)00136-2

25. Colloca M., Gupta N., Porfiri M. (2013): Tensile properties of carbon nanofiber reinforced multiscale syntactic foams. Composites Part B: Engineering, 44(1), 584-591. doi:10.1016/j.compositesb.2012.02.030

26. Saha M.C., Nilufar S. (2010): Nanoclay-reinforced syntactic foams: Flexure and thermal behavior. Polymer Composites, 31(8), 1332-1342. doi:10.1002/pc.20918

27. Wouterson E.M., Boey F.Y.C., Hu X., Wong S.C. (2007): Effect of fiber reinforcement on the tensile, fracture and thermal properties of syntactic foam. Polymer, 48, 31833191. doi:10.1016/j.polymer.2007.03.069

28. Porfiri M., Gupta N. (2009): Effect of volume fraction and wall thickness on the elastic properties of hollow particle filled composites. Composites Part B: Engineering, 40(2), 166-173. doi:10.1016/j.compositesb.2008.09.002
29. Marur P.R. (2005): Effective elastic moduli of syntactic foams. Materials Letters, 59, 1954-1957. doi:10.1016/j. matlet.2005.02.034

30. Marur P.R. (2010): Numerical estimation of effective elastic moduli of syntactic foams. Finite Elements in Analysis and Design, 46(11), 1001-1007. doi:10.1016/j. finel.2010.07.006

31. Bardella L., Stfreddo A., Ventura C., Porfiri M., Gupta Nikhil (2012): A critical evaluation of micromechanical models for syntactic foams. Mechanics of Materials, 50, 53-69. doi:10.1016/j.mechmat.2012.02.008

32. Yu M., Zhu P., Ma Y. (2013): Effects of particle clustering on the tensile properties and failure mechanisms of hollow spheres filled syntactic foams: A numerical investigation by microstructure based modeling. Materials \& Design, 47, 80-89. doi:10.1016/j.matdes.2012.12.004

33. Nguyen N.Q., Gupta N. (2010): Analyzing the effect of fiber reinforcement on properties of syntactic foams. Materials Science and Engineering: A, 527(23), 64226428. doi:10.1016/j.msea.2010.06.077

34. Patankar S.N., Das A., Kranov Y.A. (2009): Interface engineering via compatibilization in HDPE composite reinforced with sodium borosilicate hollow glass microspheres. Composites Part A: Applied Science and Manufacturing, 40(6), 897-903. doi:10.1016/j. compositesa.2009.04.016

35. Liang X., Li H.J., Yu W., Jiang X., Zhang Z.Q. (2013): Chinese J. Solid Mech. 34, 73

36. Kari S., Berger H., Rodriguez-Ramos R., Gabbert U. (2007): Computational evaluation of effective material properties of composites reinforced by randomly distributed spherical particles. Composite Structures, 77(2), 223-231. doi:10.1016/j.compstruct.2005.07.003

37. Pierard O., LLorca J., Segurado J., Doghri I. (2007): Micromechanics of particle-reinforced elasto-viscoplastic composites: finite element simulations versus affine homogenization. International Journal of Plasticity, 23(6), 1041-1060. doi:10.1016/j.ijplas.2006.09.003 